\title{
On the true nature of renormalizability in Horava-Lifshitz gravity
}

\author{
Fabio Briscese $e^{a, b *}$, Yeinzon Rodríguez ${ }^{a, c \dagger}$, Guillermo A. González ${ }^{a \ddagger}$. \\ a Escuela de Física, Universidad Industrial de Santander, \\ Ciudad Universitaria, Bucaramanga 680002, Colombia. \\ ${ }^{b}$ Istituto Nazionale di Alta Matematica Francesco Severi, \\ Gruppo Nazionale di Fisica Matematica, \\ Città Universitaria, c.a.p. 00185, Rome, Italy. \\ c Centro de Investigaciones, \\ Universidad Antonio Nariño, \\ Cra 3 Este \# 47A - 15, \\ Bogotá D.C. 110231, Colombia.
}

\begin{abstract}
We argue that the true nature of the renormalizability of Horava-Lifshitz gravity lies in the presence of higher order spatial derivatives and not in the anisotropic Lifshitz scaling of space and time. We discuss the possibility of constructing a higher order spatial derivatives model that has the same renormalization properties of Horava-Lifshitz gravity but that does not make use of the Lifshitz scaling. In addition, the state-of-the-art of the Lorentz symmetry restoration in Horava-Lifshitz-type theories of gravitation is reviewed.
\end{abstract}

The quantum gravity problem, namely the need of an unified description of all fundamental interactions in the same theoretical framework, is one of the most challenging subjects of modern physics. The most important approaches to quantum gravity are Loop Quantum Gravity, Strings, and Noncommutative Geometries (see [1] for an introductory reading), but recently a new model of gravity, the so called Horava-Lifshitz Gravity (H-LG) 2], has been proposed and it is believed to be renormalizable. H-LG has been shown to be a viable model of gravitation at cosmological and astrophysical levels $3[-6]$, and black hole thermodynamics has been also considered [710], but one of the main problems with such a model is that it implies the loss of Lorentz symmetry.

H-LG makes use of two different assumptions: the introduction of higher order spatial derivatives [44] in the lagrangian density of the gravitational field and the anisotropic Lifshitz scaling of space and time.

Due to higher order spatial derivatives, the invariance of General Relativity (GR) under diffeomorphisms $\operatorname{Diff}(M)$ is broken. Therefore, the spacetime $M$ is assumed to be a codimension-one foliable and differentiable manifold and the theory is invariant under the group of foliation preserving diffeomorphisms $\operatorname{Diff}_{F}(M)$, which leave the spatial derivatives invariant. We stress that the invariance of the theory under $\operatorname{Diff}_{F}(M)$ does not imply the Lifshitz scaling.

Higher derivative renormalizable theories of gravitation have been first presented in [11] which are invariant under GR diffeomorphysms Diff $(M)$. The main problem here is that they contain a physical ghost (a state of negative norm) and therefore they violate the unitarity. Recently, a new class of higher derivative theories has

\footnotetext{
*E-mail: briscese.phys@gmail.com

${ }^{\dagger}$ E-mail: yeinzon.rodriguez@uan.edu.co

‡E-mail: guillego@uis.edu.co
}

been presented that turns out to be both renormalizable and ghost-free [12 16]. The price to pay here is that the theory should contain an infinite number of derivatives to avoid the loss of unitarity. However, H-LG is naturally ghost-free [3] since it contains higher order space derivatives but no higher order time derivatives. Moreover, the renormalizability condition only requires space derivatives of finite order, therefore $\mathrm{H}-\mathrm{LG}$ is simpler than an infinite order theory. Despite of these advantages, there is an apparently strong problem in H-LG: the loss of diffeomorphism invariance and then the loss of Lorentz symmetry in flat spacetime as discussed in [17 20].

In this Letter we argue that the true nature of the renormalizability of H-LG lies in the presence of higher order spatial derivatives in the lagrangian density of the gravitational field and not in the anisotropic Lifshitz scaling of space and time.

The power counting renormalizability of H-LG is deduced by an argument based on dimensional analysis 2, 3] which makes use of the Lifshitz scaling. As we will show, such a dimensional analysis is incorrect and the Lifshitz scaling results to be unnecessary for the renormalizability of the theory. Therefore the renormalizability of H-LG would be due uniquely to the presence of higher order spatial derivatives in the lagrangian density of the gravitational field and not due to the Lifshitz scaling. In fact, because of the higher order derivatives, the graviton propagator goes to zero more rapidly than $1 / k^{2}$ at high wave numbers $k$, ensuring the convergence of Feynman diagrams.

We stress that Lifshitz scaling and higher order spatial derivatives are two distinct features of H-LG which are not equivalent. As an example of this fact, we will introduce in (15) a new gravitational model with higher order spatial derivatives but that does not make use of the Lifshitz scaling. In particular this model has the same gravitational lagrangian density of H-LG and, therefore, has the same graviton propagators and renormalization properties of H-LG. Conversely, assuming Lifshitz scal- 
ing but no higher order derivatives, it does not lead to a renormalizable theory since in that case the graviton propagator scales as $1 / k^{2}$ as in GR. These examples just confirm the fact that the Lifshitz scaling is not necessary for the renormalizability of H-LG-type theories of gravitation.

We remark that the new model (15) is invariant under Dif $f_{F}(M)$ since it contains higher order spatial derivatives that break the Diff $(M)$ invariance of GR, but it does not use the Lifshitz scaling. However, the breaking of $\operatorname{Diff}(M)$ implies the loss of Lorentz invariance. For that reason, we will discuss the possibility of recovering the Lorentz symmetry in flat spacetime background in H-LG-type theories in the Standard Model (SM) particle sector at energies below the Planck scale.

In what follows, we will first resume the main properties of H-LG and show that the power counting renormalization argument introduced in [2] is incorrect. Then, we will argue that the renormalization of the model is due to the presence of higher order spatial derivatives and not due to the Lifshitz sclaing. We will introduce a new higher derivative model that has the same renormalization properties of H-LG but that does not make use of the Lifshitz scaling. Finally we will discuss the Lorentz symmetry restoration in Horava-Lifshitz-type theories as known in the literature.

Differently from GR, H-LG consider space and time as fundamentally different quantities. In fact H-LG assumes an anisotropic scaling, or Lifshitz scaling, of space and time given by

$$
t \rightarrow b^{z} t, \quad \vec{x} \rightarrow b \vec{x}
$$

where $z$ is the dynamical critical exponent of the theory. Eq. (1) is equivalent to fix the dimensions of time and space coordinates as $[t]=[x]^{z}$. Defining a formal symbol $p$ having dimension of momentum, and taking $\hbar=1$, one has $[t]=[p]^{-z}$ and $[x]=[p]^{-1}$ (see $\left.[21,22]\right)$ and therefore one has $[t]=-z$ and $[x]=-1$ in momentum units $[p]$. Here and below, all the dimensions will be expressed in momentum units $[p]$. This scaling law is believed to imply the renormalizability of the theory [2, 3].

The fundamental symmetry of the theory is the invariance under $\operatorname{Dif} f_{F}(M)$, that in a local reference frame can be expressed as

$$
t^{\prime}=t^{\prime}(t), \quad \vec{x}^{\prime}=\vec{x}^{\prime}(\vec{x}, t) .
$$

Note that the invariance under $\operatorname{Diff}_{F}(M)$ does not imply the Lifshitz scaling law (1). Moreover, one can always perform the transformation $t^{\prime}=t^{1 / z}$ and $x^{\prime}=x$ allowed by the $D i f f_{F}(M)$ symmetry, which reduces to isotropic scaling of space and time coordinates $\left[t^{\prime}\right]=\left[x^{\prime}\right]=-1$. This gives an intuition on the fact that the Lifshitz scaling cannot be relevant for the renormalizability of the theory.

Also note that Lorentz symmetry is not a symmetry of the theory since it is not included in $D$ if $f_{F}(M)$.

Due to Lifshitz scaling the speed of light now has $z$ dependent dimensions in momentum units $[c]=[d x / d t]=$ $z-1[21,22]$. Therefore one can not simply take $[c]=1$, but it is convenient to explicitly express the dependence on $c$ in all physical quantities. To construct the lagrangian of the theory in $\mathrm{D}+1$ dimensions, one can write the $\mathrm{D}+1$-dimensional metric tensor $g_{i j}^{(D+1)}$ in the ADM formalism [23] as

$$
d s^{2}=-N^{2}(c d t)^{2}+2 N_{\alpha} c d t d x^{\alpha}+g_{\alpha \beta}^{(D)} d x^{\alpha} d x^{\beta},
$$

where Greek indices run from 1 to $D$ and Latin indices from 0 to $\mathrm{D}$, and $N=g_{00}^{(D+1)}, N_{\alpha}=g_{0 \alpha}^{(D+1)}$ and $g_{\alpha \beta}^{(D)}=g_{\alpha \beta}^{(D+1)}$ are the lapse, the shift and the Ddimensional spatial metric tensor respectively. Due to the invariance under $\operatorname{Dif} f_{F}(M)$, the lapse $N(t)$ is required to be a function of time only.

Note that the GR time variable $x^{0}=c t$ has dimensions $\left[x^{0}\right]=-1$ in momentum units. The GR prescription implies the use of the variable $x^{0}$ instead of $t$ in all physical quantities. Of course one can always write the line element as $d s^{2}=-N^{2} d t^{2}+2 N_{\alpha} d t d x^{\alpha}+g_{\alpha \beta}^{(D)} d x^{\alpha} d x^{\beta}$. However, in such a case, the dimension of $c$ would be absorbed in the dimensions of the lapse and shift and one would have $[N]=\left[N_{\alpha}\right]=z-1$ so the physics would remain unchanged.

The action of the theory is [3]

$$
S_{H-L G} \equiv \frac{2}{k^{2}} \int c d t d^{D} x N \sqrt{-g^{(D)}}\left(L_{H-L G}+L_{S M}\right)
$$

where $L_{H-L G}$ is the H-LG lagrangian density and $L_{S M}$ is the usual SM lagrangian density. $L_{H-L G}$ contains the higher order spatial derivatives that should ensure the renormalizability of the theory. Its explicit form is given by [3]

$$
L_{H-L G} \equiv K^{\alpha \beta} K_{\alpha \beta}-\lambda K^{2}+R^{(D)}-2 \Lambda+I_{z=2}+I_{z=3},
$$

where $\lambda$ and $\Lambda$ are constants, $R^{(D)}$ is the Ricci scalar constructed with $g_{\alpha \beta}^{(D)}, K_{\alpha \beta}$ is the extrinsic curvature defined as

$$
K_{\alpha \beta} \equiv \frac{1}{2 N}\left(\partial_{c t} g_{\alpha \beta}^{D}-D_{\alpha} N_{\beta}-D_{\beta} N_{\alpha}\right)
$$

$k \equiv K_{\alpha}^{\alpha}, D_{\alpha}$ is the covariant derivative constructed with $g_{\alpha \beta}^{(D)}$ and

$$
\begin{aligned}
I_{z=3} \equiv & c_{1} D_{\alpha} R_{\beta \gamma}^{(D)} D^{\alpha} R^{(D) \beta \gamma}+c_{2} D_{\alpha} R^{(D)} D^{\alpha} R^{(D)}+ \\
& +c_{3} R_{\beta}^{(D) \alpha} R_{\alpha}^{(D) \gamma} R_{\gamma}^{(D) \beta}+c_{4} R^{(D)} R_{\beta}^{(D) \alpha} R_{\alpha}^{(D) \beta}+ \\
& +c_{5} R^{(D) 3}, \\
I_{z=2} \equiv & c_{6} R_{\alpha}^{(D) \beta} R_{\beta}^{(D) \alpha}+c_{7} R^{(D) 2} .
\end{aligned}
$$

H-LG has a scalar $\zeta$ and a transverse traceless tensor $h_{\alpha \beta}$ physical degrees of freedom defined by $g_{\alpha \beta}=(1+$ 
$2 \zeta) \delta_{\alpha \beta}+h_{\alpha \beta}$, whose dispersion relations are

$$
\begin{array}{r}
\omega_{\zeta}^{2}=\frac{\lambda-1}{3 \lambda-1}\left(\frac{k^{6}}{M_{s}^{4}}+\kappa_{s} \frac{k^{4}}{M_{s}^{2}}-k^{2}\right), \\
\omega_{h}^{2}=\frac{k^{6}}{M_{t}^{4}}+\kappa_{t} \frac{k^{4}}{M_{t}^{2}}+k^{2},
\end{array}
$$

where [3]

$$
\begin{aligned}
M_{s}^{-4} & \equiv-2\left(3 c_{1}+8 c_{2}\right) M_{P}^{-2}, \\
\kappa_{s} M_{s}^{-2} & \equiv-2\left(3 c_{6}+8 c_{7}\right) M_{P}^{-2}, \\
M_{t}^{-4} & \equiv-2 c_{1} M_{P}^{-2} \\
\kappa_{t} M_{t}^{-2} & \equiv-2 c_{6} M_{P}^{-2}
\end{aligned}
$$

and $M_{P}$ is the reduced Planck mass. We mention that the scalar degree of freedom $\zeta$ does not decouple in the low energy limit but it can be eliminated by requiring local scale invariance as in shape dynamics [24].

Comparing Eq. (4) with the Einstein-Hilbert action of GR, we can see that the GR diffeomorphism invariance $\operatorname{Diff}(M)$ is broken at two different levels. First, it is broken at the level of the density lagrangian $L_{H-L G}$, since this is constructed with quantities explicitly invariant under $\operatorname{Diff}_{F}(M)$; second, it is broken at the level of the $\mathrm{D}+1$ spacetime measure $c d t d^{D} x N \sqrt{-g^{D}}$.

In Ref. 2], the assumption of the Lifshitz scaling law (11) is motivated since it would lead to the power counting renormalizability of the theory. This conclusion is based on the following dimensional argument. First of all note that in [2] the author uses Eqs. (3)-(5) with $c=1$ ignoring the speed of light $c$ and its dimensions. This implies that the dimensions of $c$ are included in the dimensions of other physical quantities. For example in [2] the author uses $[N]=0,\left[N_{\alpha}\right]=z-1$ (Eq. (2.4)) and $\left[K_{i j}\right]=z$. By means of such a dimensional analysis, and requiring the dimensionless of the action (4), one would conclude that the dimension of the constant $k$ is $(z-D) / 2$ (Eq. (21) in [2]). This would lead to the conclusion that the dimension of $k$ would be non negative for $z \geq D$ and that, therefore, the theory would be power counting renormalizable.

Unfortunately, this dimensional argument is not correct and this is evident if one includes explicitly the speed of light $c$ in Eqs. (3)-(5) as we did. From Eq. (3), it is immediate to recognize that the lapse and the shift should have the same dimension $[N]=\left[N_{\alpha}\right]=0$ since $\left[d s^{2}\right]=-2$. This also implies that $\left[L_{H-L G}\right]=2\left[K_{i j}\right]=2$. Requiring the dimensionless of the action $\left[S_{H-L G}\right]=0$ and using Eq.(4) with the correct $c d t$ factor in the integral, one concludes that the constant $k$ has dimension $[k]=(1-D) / 2$ independently of $z$ as it should be. We stress again that the difference between the two dimensional analysis lies on the fact that in Ref. [2] the dependence on $c$ is not considered explicitly. We also stress that, as noted before, even if one does not consider explicitly the light speed $c$, the dimensions of the lapse and shift functions should be the same $[N]=\left[N_{\alpha}\right]=z-1$ and, therefore, Eq. (2.4) of [2] is not correct. Using the correct dimensions $z-1$ of the lapse and shift functions, one again obtains the correct dimension $[k]=(1-D) / 2$ which is independent of $z$.

Based on such observations, one can conclude that the renormalizability of H-LG cannot be motivated by the dimensional analysis performed in [2]. Whereas the power counting argument used in 2] has been shown to be incorrect, the renormalizability of H-LG remains to be demonstrated rigorously. We notice that in 25], the renormalizability of H-LG is related to that of topologically massive gravity but, as the authors remark, the latter is not strictly proven.

Therefore, we conclude that the Lifshitz scaling has no any role in the renormalizability of H-LG since the correct dimensional analysis has been shown to be independent of the dynamical critical exponent $z$. We think that the true nature of the renormalizability of H-LG lies in the presence of higher derivative terms in the lagrangian and not in the scaling law (1).

In fact, renormalizability seems to be a feature of higher derivative theories [11 16], due to the fact that the presence of higher order derivatives implies that the graviton propagator goes to zero more rapidly than $1 / k^{2}$ at high wave numbers $k$. However, in order to be unitary, such higher derivative theories should contain an infinite number of derivatives 12 16]. The advantage of H-LG with respect to higher order derivative theories is that it is naturally ghost-free [3] and includes a limited number of space derivatives but with the handicap of losing Lorentz symmetry in flat spacetime.

Let us introduce a new model with higher spatial derivatives that has the same renormalization properties of H-LG but that does not make use of the Lifshitz scaling law (11). This example explicitly shows that the Lifshitz scaling is unnecessary for the renormalizability. Moreover, it can can be of interest since, as we will see, it is more conservative than H-LG since it does not make use of the Lifshitz scaling and uses the same volume element of GR. This new model is only introduced here but its main properties deserve further study.

Let us use the usual scaling of time and space $[t]=$ $[x]=-1$. This legitimates the use of natural units with $c=1$. Moreover, in order to be conservative, we can break the diffeomorphism invariance of GR only at the level of the gravitational lagrangian density and take the same $\mathrm{D}+1$ dimensional measure and SM lagrangian density as in GR. We restrict to the case $\mathrm{D}=3$ and write the action for such a new theory as

$$
S \equiv \frac{2}{k^{2}} \int d^{4} x \sqrt{-g^{(3+1)}}\left(L_{H-L G}+L_{S M}\right),
$$

where $L_{H-L G}$ is the same as in (5) and $d^{4} x=d t d^{3} x$. Taking $\lambda=1, k^{2}=32 \pi G$, and $\Lambda=0$, the action (15) describes GR plus higher order derivatives [3]. For simplicity, we use the same lagrangian density $L_{H-L G}$ as $\mathrm{H}-$ LG since it contains higher order spatial derivatives that should ensure the renormalizability of the theory, but it 
is clear that one can make a different choice, e.g. adding Dif $f_{F}(M)$-invariant terms containing even higher order spatial derivatives. Also note that (15) reduces to (4) in the synchronous gauge when $D=3$.

Since in Eq. (15) we take the same lagrangian density $L_{H-L G}$ of $\mathrm{H}-\mathrm{LG}$, expanding the gravitational field around a flat spacetime background gives the same kinetic term of H-LG. Therefore, one has the same scalar $\zeta$ and traceless tensor $h_{\alpha \beta}$ gravitational degrees of freedom and the same dispersion relations (9) and (10) as in H-LG and then the same propagators for the $\zeta$ and $h_{\alpha \beta}$ fields. This implies that (15) has the same renormalization properties of H-LG. However the renormalizability of (15), as well as that of H-LG, remains to be proved rigorously.

We remark that in the model (15), the parameter $z=3$ is no longer the dynamical critical exponent of the theory (which does not assume Lifshitz scaling) but is simply related to the order $2 z=6$ of spatial derivatives. Therefore, the UV behavior $\sim k^{6}$ of the graviton propagators is due to higher order derivatives and is not related to the Lifshitz scaling.

Of course, the new quantum gravity framework described by (15) should be tested in the pure gravitational sector, meaning that one should study the behavior of gravity at cosmological and astrophysical levels. These topics deserve further study.

We remark as well that in this new theory one has to deal with the same SM Lorentz restoration problem of H-LG [17]. Therefore, here we discuss Lorentz symmetry restoration in H-LG-type gravities.

It is evident from (4) that, if the gravitational field is turned off, the residual SM action is Lorentz invariant. Let us consider SM particles in a flat background spacetime plus quantum gravitational perturbations. Note that SM particle propagators are Lorentz invariant at tree level, but graviton propagators are not since the lagrangian density of the gravitational field is explicitly not a Lorentz invariant. Of course, loop corrections will contain graviton propagators and, therefore, will break Lorentz invariance of SM particle propagators.

Since in (4) SM particles are minimally coupled to gravity, this implies that, as in GR, interactions between SM particles and gravitons are expected to be Planck scale suppressed. Therefore one expects that Lorentz symmetry breaking loop corrections to SM propagators are Planck scale suppressed and Lorentz symmetry is safe.

Unfortunately, this is not the case since, as shown in Ref. [17], one loop Lorentz symmetry breaking effects are Planck scale suppressed but coupled to quadratically divergent diagrams and results to be $\propto\left(\Lambda_{H L} / M_{P}\right)^{2} \Lambda_{U V}^{2}$ where $\Lambda_{H L} \sim c_{1}, c_{2}, \ldots, c_{5}$, is the Lorentz symmetry breaking scale and $\Lambda_{U V}$ is the ultraviolet (UV) cutoff. That means that Lorentz symmetry breaking in the SM sector is never negligible in this picture. In Ref. [17], the authors propose to introduce higher derivative terms as $K_{\alpha \beta} \Delta K^{\alpha \beta}$ or $\nabla_{\alpha} K^{\alpha \beta} \nabla^{\gamma} K_{\beta}^{\gamma}$ in order to regularize such a quadratically divergence, but this possibility has not been yet completely explored.

Lorentz symmetry restoration is a hard problem for H-LG-type models. In fact, if Lorentz symmetry is broken in the SM sector below the Planck scale, the SM particle propagators would not be Lorentz invariant and the Minkowskian energy-momentum "dispersion" relation would be deformed below the Planck scale. Such dispersion relation deformations have been shown to be testable in some context up to the Planck scale by using astrophysical data 26 41] or in cold atoms recoil frequency experiments [42, 43] and, therefore, a subPlanckian Lorentz symmetry breaking could rule out the model.

The scope of this Letter is to generate a debate about the true nature of the renormalizability of H-LG. What is important here is to show that the anisotropic scaling law (11) is not necessary to ensure the renormalization of H-LG. We argued that such renormalizability would be due only to higher order derivatives and not due to the Lifshitz scaling (11). We have introduced a new higher spatial derivatives model that does not make use of the Lifshitz scaling an that does not break $\operatorname{Diff}(M)$ in the spacetime measure. This model results more conservative but has the same renormalization properties of $\mathrm{H}$ LG. Finally, we have discussed the Lorentz invariance restoration problem in H-LG-type theories as known in the literature.

Acknowledgments: We thank A. Marcianó, D. H. Lyth, S. Mukohyama, M. Sasaki, and M. de Llano for useful discussions during the edition of this Letter. F.B. is a UIS postdoctoral fellow. Y.R. is supported by DIEF de Ciencias (UIS) grant number 5177.
[1] L. Smolin, Three roads to quantum gravity, London, UK: Weidenfeld \& Nicolson (2000).

[2] P. Hořava, Phys. Rev. D 79, 084008 (2009).

[3] S. Mukohyama, Class. Quantum Grav. 27, 223101 (2010).

[4] K. Izumi and S. Mukohyama, Phys. Rev. D 84, 064025 (2011).

[5] A. E. Gumrukcuoglu, S. Mukohyama, and A. Wang, Phys. Rev. D 85, 064042 (2012).
[6] P. Hořava and C. M. Melby-Thompson, Phys. Rev. D 82, 064027 (2010).

[7] R. Biswas and S. Chakraborty, Gen. Rel. Grav. 4341 (2011).

[8] W. Janke, D. A. Johnston, and R. Kenna, J. Phys. A 43, 425206 (2010).

[9] Q.-J. Cao, Y.-X. Chen, and K.-N. Shao, Phys. Rev. D 83, 064015 (2011).

[10] H. Quevedo, A. Sánchez, S. Taj, and A. Vázquez, J. Phys. 
A 45, 055211 (2012).

[11] K. S. Stelle, Phys. Rev. D 16, 953 (1977).

[12] J. W. Moffat, Eur. Phys. J. Plus 126, 43 (2011).

[13] E. T. Tomboulis, arXiv:hep-th/9702146

[14] L. Modesto, arXiv:1107.2403 [hep-th].

[15] L. Modesto, arXiv:1206.2648 [hep-th].

[16] L. Modesto, arXiv:1202.3151 [hep-th].

[17] M. Pospelov and Y. Shang, Phys. Rev. D 85, 105001 (2012).

[18] C. Bogdanos and E. N. Saridakis, Class. Quantum Grav. 27, 075005 (2010).

[19] F.S. Bemfica and M. Gomes, Phys. Rev. D 84, 084022 (2011).

[20] F.S. Bemfica and M. Gomes, arXiv:1111.5779 [hep-th].

[21] M. Visser, Phys. Rev. D 80, 025011 (2009).

[22] S. Weinfurtner, T.P. Sotiriou, and M. Visser, J. Phys. Conf. Ser. 222, 012054 (2010).

[23] R. Arnowitt, S. Deser, and C. W. Misner, Gravitation: an introduction to current research, New York, USA: Wiley (1962).

[24] H. Gomes, S. Gryb, and T. Koslowski, Class. Quantum Grav. 28, 045005 (2011).

[25] D. Orlando and S. Reffert, Class. Quantum Grav. 26, 155021 (2009).

[26] C. Charmousis, G. Niz, A. Padilla, and P. M. Saffin, JHEP 0908, 070 (2009).

[27] R. Iengo, J. G. Russo, and M. Serone, JHEP 0911, 020
(2009).

[28] S. Mukohyama, K. Nakayama, F. Takahashi, and S. Yokoyama, Phys. Lett. B 679, 6 (2009).

[29] Z. Xiao and B.-Q. Ma, Phys. Rev. D 80, 116005 (2009).

[30] L. Shao, Z. Xiao, and B.-Q. Ma, Astropart. Phys. 33, 312 (2010).

[31] G. Amelino-Camelia, et. al., Nature 393, 763 (1998).

[32] B. E. Schaefer, Phys. Rev. Lett. 82, 4964 (1999).

[33] S. D. Biller et. al., Phys. Rev. Lett. 83, 2108 (1999).

[34] T. Kifune, Astrophys. J. Lett. 518, L21 (1999).

[35] G. Amelino-Camelia, Nature 408, 661 (2000).

[36] A. A. Abdo et. al., Science 323, 1688 (2009).

[37] J. Ellis, N. E. Mavromatos, and D. V. Nanopoulos, Phys. Lett. B 674, 83 (2009).

[38] G. Amelino-Camelia and L. Smolin, Phys. Rev. D 80, 084017 (2009).

[39] A. A. Abdo et al., Nature 462, 331 (2009).

[40] G. Amelino-Camelia, Nature 462, 291 (2009).

[41] G. Amelino-Camelia, L. Gualtieri, and F. Mercati, Phys. Lett. B 686, 283 (2010).

[42] G. Amelino-Camelia, C. Lämmerzahl, F. Mercati, and G. M. Tino, Phys. Rev. Lett. 103, 171302 (2009).

[43] F. Mercati et. al., Class. Quantum Grav. 27, 215003 (2010).

[44] With spatial derivatives we mean covariant derivatives constructed with the spatial components of the metric tensor. 